\title{
Increased decision thresholds trigger extended information gathering across the compulsivity spectrum
}

\author{
Tobias U. Hauser [1, ${ }^{1,2}$, Michael Moutoussis ${ }^{1,2}$, NSPN Consortium, Peter Dayan ${ }^{3}$ and Raymond J. Dolan ${ }^{1,2}$
}

\begin{abstract}
Indecisiveness and doubt are cognitive phenotypes of compulsive disorders, including obsessive-compulsive disorder. Little is known regarding the cognitive mechanisms that drive these behaviours across a compulsivity spectrum. Here, we used a sequential information gathering task to study indecisiveness in subjects with high and low obsessivecompulsive scores. These subjects were selected from a large population-representative database, and matched for intellectual and psychiatric factors. We show that high compulsive subjects sampled more information and performed better when sampling was cost-free. When sampling was costly, both groups adapted flexibly to reduce their information gathering. Computational modelling revealed that increased information gathering behaviour could be explained by higher decision thresholds that, in turn, were driven by a delayed emergence of impatience or urgency. Our findings show that indecisiveness generalises to a compulsivity spectrum beyond frank clinical disorder, and this behaviour can be explained within a decision-theoretic framework as arising from an augmented decision threshold associated with an attenuated urgency signal.
\end{abstract}

\section{Introduction}

A tradeoff between certainty and the time spent on option evaluation is a crucial, and non-trivial aspect of decision making ${ }^{1-3}$. Spending too little time on an important decision (e.g., whom to marry) can be highly deleterious, as can spending too much time making relatively unimportant decisions (e.g., where to buy lunch).

Patients with obsessive-compulsive disorder (OCD) appear to suffer the latter affliction, and can be described as both indecisive and intolerant of uncertainty ${ }^{4,5}$. Indeed, many symptoms can be thought of in terms of extended information gathering behaviour that is the complement of OCD subjects' need for certainty (e.g., checking that all

\footnotetext{
Correspondence: Tobias U. Hauser (t.hauser@ucl.ac.uk)

${ }^{1}$ Wellcome Trust Centre for Neuroimaging, University College London, London WC1N 3BG, United Kingdom

${ }^{2}$ Max Planck UCL Centre for Computational Psychiatry and Ageing Research, London WC1B 5EH, United Kingdom

Full list of author information is available at the end of the article

Dayan Peter and Raymond J. Dolan contributed equally to this work.
}

windows are closed). Despite a rich symptomatic description in the literature there is a dearth of experimental studies that decompose this behaviour. Most studies support a link between OCD and increased information gathering behaviour ${ }^{6-10}$, although not unequivocally $y^{11-13}$. In a recent computational study, we showed that adolescent patients with OCD express increased information gathering due to an increased decision threshold and altered subjective sampling costs ${ }^{7}$.

A fundamental problem in many clinical studies is the observation that patients often suffer additional comorbidities, notably (sub-)clinical levels of depression and anxiety as well as potential influences from current or past medication. There is also concern in relation to the explanatory utility of categorical psychiatric diagnoses ${ }^{14,15}$ that has led to a re-conceptualisation in terms of psychiatric dimensions ${ }^{16}$. In this latter framework patients with OCD represent those at an extreme of a compulsivity spectrum while allowing for the presence of considerable 
Table 1 Subject characteristics

\begin{tabular}{llll}
\hline & Low compulsive group & High compulsive group & \\
\hline Age* & $21.40 \pm 2.52$ & $20.75 \pm 2.34$ & $\mathrm{t}(38)=.85, p=.403$ \\
Gender (f/m)* & $13 / 7$ & $14 / 6$ & $\mathrm{x}(1)=.114, p=.736$ \\
Handedness (r/l) & $16 / 4$ & $16 / 4$ & $\mathrm{x}(1)=.0, p=1.00$ \\
IQ (WASI total) & $115.6 \pm 10.9$ & $115.4 \pm 9.8$ & $\mathrm{t}(38)=.06, p=.952$ \\
PI-WSUR tota|* & $5.3 \pm 4.0$ & $50.2 \pm 18.3$ & $\mathrm{t}(38)=10.74, p<.001$ \\
MFQ* & $19.1 \pm 8.9$ & $19.4 \pm 11.7$ & $\mathrm{t}(38)=.07, p=.942$ \\
RCMAS total* & $20.7 \pm 10.1$ & $18.7 \pm 10.7$ & $\mathrm{t}(38)=-.61, p=.545$ \\
BDI-II total & $6.1 \pm 4.2$ & $8.7 \pm 7.1$ & $\mathrm{t}(38)=1.41, p=.166$ \\
STAI (trait) & $38.3 \pm 6.5$ & $41.4 \pm 11.3$ & $\mathrm{t}(38)=1.05, p=.302$ \\
STAI (state) & $33.5 \pm 6.1$ & $34.0 \pm 9.4$ & $\mathrm{t}(38)=.20, p=.843$ \\
BIS & $58.30 \pm 6.87$ & $59.04 \pm 9.74$ & $\mathrm{t}(38)=-.28, p=.782$ \\
Intolerance of uncertainty & $48.75 \pm 15.14$ & $58.80 \pm 15.87$ & $\mathrm{t}(38)=-2.05, p=.047$ \\
FMPS total & $98.02 \pm 16.21$ & $103.02 \pm 17.35$ & $\mathrm{t}(38)=-0.94, p=.353$ \\
\hline
\end{tabular}

Subjects were recruited from a population-based database so that groups differed maximally on the compulsivity spectrum (PI-WSUR) ${ }^{21}$, but were matched for age, gender, depression (MFQ) $)^{22}$ and anxiety (RCMAS) ${ }^{24}$. The groups did not differ in depression (BDI-II) ${ }^{27}$, anxiety (STAI) ${ }^{26}$, impulsivity $(\mathrm{BIS})^{28}{ }^{2}$, handedness ${ }^{30}$ or intellectual abilities (WASI) ${ }^{29}$, as assessed on the day of the experiment. Groups differed in their intolerance of uncertainty ${ }^{33}$, but not in perfectionism (FMPS) ${ }^{46}$. (mean \pm SD); ${ }^{*}$ data used for recruiting participants

compulsivity heterogeneity within an otherwise 'healthy' population.

We sought to examine whether excessive information gathering behaviour was a marker of such a compulsivity spectrum rather than simply the expression of a categorical disease state. We recruited forty young adults from a large population-representative sample from whom we had collected psychiatric and other health-related information. We followed a targeted recruitment approach, in which we selected non-clinical subjects scoring either high or low on an obsessive-compulsive symptom scale, but matching these groups on other (psychiatric) dimensions, such as mood, anxiety, age, gender, and intellectual abilities. This allowed us to overcome limitations in patient studies, such as those arising out of comorbidities or medication usage. Using a sequential information gathering task, we show that high compulsive subjects express similar behavioural and computational features as patients with OCD, consistent with the general idea of a compulsivity spectrum where pathology represents an extreme.

\section{Materials and methods \\ Subjects}

The goal of this study was to compare information gathering in subjects who differed in obsessivecompulsive symptoms (subsequently called 'compulsivity' for short, but this is not intended to imply an exclusion of obsessions), but were comparable on other psychiatric traits, in particular in relation to symptoms of depression and anxiety. This is important because a high comorbidity between OCD, depression and anxiety often renders a dissociation difficult for classic patient studies [17]. We thus used a large population-representative sample of young people in London and Cambridge (UCHANGE study; $N=2409$; www.nspn.org.uk ${ }^{18-20}$ ), from whom we had collected questionnaire and health-related information. From this database, we recruited 20 adult subjects with low $(21.40 \pm 2.52$ years $)$ and 20 adults with high compulsivity scores $(20.75 \pm 2.34$ years; group details see Table 1). Importantly, the low compulsive subjects were specifically selected so as to match the high compulsives on anxiety and depression scores, which allowed us to determine whether information gathering biases were specific to variation in the compulsivity spectrum.

As an index of compulsivity, we used the total score of the revised Padua Inventory questionnaire (PI-WSUR $)^{21}$. The PI-WSUR is an established questionnaire for assessing obsessive-compulsive traits with a high test-retest reliability and internal consistency ${ }^{21}$. The subjects with high compulsive scores were in the $91.62 \pm 5.83$ percentile of the U-CHANGE populations' PI-WSUR distribution, whereas the low compulsive group scored on the $27.29 \pm$ 16.55 percentile of this distribution.

To match groups for depressive mood, we recruited subjects based on the Mood and Feelings Questionnaire $(\mathrm{MFQ})^{22}$, which has a high internal consistency and can be used as a screening for depression ${ }^{23}$. As a recruitment measure of anxiety, we used the Revised Children's Manifest Anxiety Scale (RCMAS) ${ }^{24}$, again known to have 


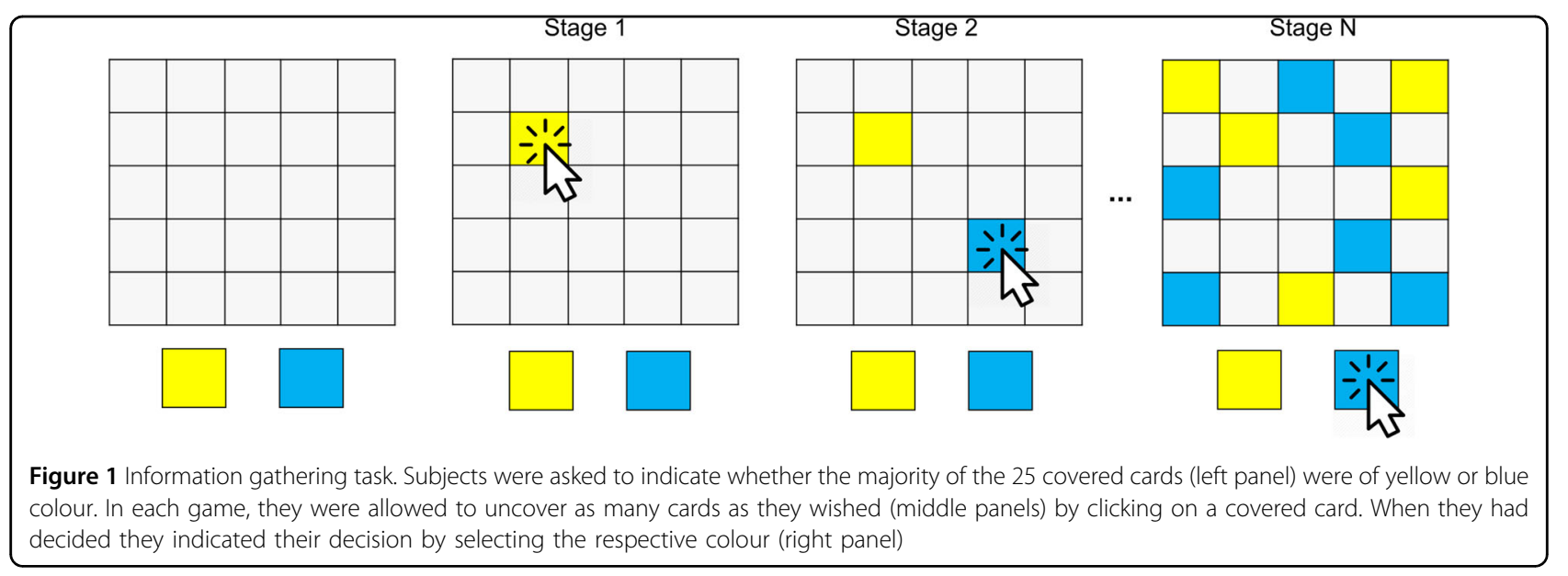

good psychometric properties ${ }^{25}$. The recruited groups scored on both questionnaires well within the normal range of the population (MFQ: low compulsivity: $31.53 \pm$ 13.64 percentile, high compulsivity: $30.13 \pm 16.18$; RCMAS: low compulsivity: $31.63 \pm 12.06$, high compulsivity: $28.71 \pm 14.47$ ), thus not being either particularly low or high in depressive and anxiety symptoms.

At the day of the assessment, we complemented these measurements with additional questionnaires of anxiety and depression that were specifically developed for $>18$ year olds, because both MFQ and RCMAS were primarily validated in children and adolescents. We thus collected the State and Trait Anxiety Inventory (STAI) ${ }^{26}$ and Beck Depression Inventory II (BDI-II) ${ }^{27}$. In addition, we collected further measures, such as impulsivity (Barratt Impulsiveness Scale, BIS) ${ }^{28}$, intelligence (matrix and vocabulary subtests of the Wechsler Abbreviated Scale of Intelligence, WASI $)^{29}$, and handedness ${ }^{30}$. The groups differed in none of the measures (full details in Table 1), ensuring that a difference in compulsivity is the key discriminating feature between the groups.

Moreover, subjects were only recruited if they fulfilled the following additional inclusion criteria: no neurological or psychiatric diagnosis (self-reported screening question), over 18 years, living in London, absence of colour blindness. Data from these subjects has previously been reported, investigating a different task collected on the same occasion ${ }^{20}$. The study was approved by the UCL research ethics committee (No. 6218/001) and all subjects gave written informed consent. Subjects received monetary compensation for their participation, but this did not depend on the performance in the reported task.

\section{Task}

The subjects performed a paradigm based on the 'information sampling task ${ }^{1711,31,32}$ (Fig. 1). In each game, subjects saw 25 covered cards (depicted by gray squares). Each of these cards could be uncovered using the computer mouse, to reveal one of two colours. In every game subjects were instructed to indicate whether they considered the majority of cards to be blue or yellow (the colours actually varied between games, the nomenclature here is used for simplicity). They were allowed to uncover as many cards as needed (a short delay of $250 \mathrm{~ms}$ was introduced between opening the tiles), without restriction on the time spent on the task. Once they felt 'certain enough' (detailed instructions are provided in Supplementary Information) they indicated their final decision by selecting the respective colour. After their decision, a short feedback screen $(1000 \mathrm{~ms})$ provided information about how many points were won (current and total points), and the next game followed immediately. Subjects had to open at least one card prior to deciding, but there was no maximum, so that subjects were allowed to sample all 25 cards possible before deciding.

We implemented two different reward schemes (the order of presentation of the schemes was kept constant across subjects). In the first set of 10 games ('fixed' condition), subjects won or lost 100 points by declaring the correct or incorrect colour, irrespective of the number of cards they uncovered. Then, in the second set of 10 games ('decreasing' condition), the potential win decreased as a function of sampling. The potential win started at 250 points and decreased by 10 points for every card that was uncovered (e.g., a correct decision after 4 opened cards would provide $250-4 * 10=210$ points). Subjects lost 100 points for a wrong decision, irrespective of the number of uncovered cards. Before the start of the fixed condition, subjects performed one practice game to familiarise themselves with the task. Details about the sequences shown are provided in the Supplementary Information.

\section{Behavioural analysis}

We analysed performance in this task using repeatedmeasures ANOVAs with within-subject factor condition (fixed, decreasing) and between-subject factor group 
(high, low compulsives). These analyses were complemented by independent-sample $t$-tests.

Based on previous findings that patients with OCD sampled more in the fixed condition and won more points $^{7}$, a priori we focused our analyses on performance differences (esp. number of draws, points won) between the groups in the fixed condition. In addition, we explored how information gathering in the fixed condition was related to a questionnaire-based report of intolerance of uncertainty ${ }^{33}$ and whether this additionally explains information gathering, over and above the compulsivity group. Effect size metrics (partial eta-squared $\eta_{p}{ }^{2}$, Cohen's d) and 95\% confidence intervals $(\mathrm{CI})$ are reported where appropriate.

\section{Computational modelling}

To understand the cognitive processes that were driving the behavioural differences, we fitted a previously developed Bayesian computational model ${ }^{7,34}$. A description of the model, the data fitting procedure and model comparison can be found in the supplementary material. In the best-fitting model, subjects form a (Bayesian) belief about which of the colours is more likely to form a majority based on the cards they have unveiled so far (i.e., 'how likely is it that there are 13 or more yellow cards?'). Subjects then use this belief to arbitrate whether to sample more cards (non-deciding) or to decide in favour of one of the two colours. This arbitration is formed by computing state-action values $(Q \text {-values })^{35}$ that indicate the worth of taking each possible action. The $Q$-values for choosing the colours are computed based on the belief about whether a particular colour forms a majority, weighted by the outcomes of choosing the (in-)correct colour. The $Q$-value for non-deciding consists of two factors. One is the belief about how certain one will be if one continues with sampling (i.e., weighted $Q$-values of future states, computed using backwards induction). The second factor is a subjective cost per step (or urgency signal $^{36-39}$ ) that promotes earlier decisions. The ultimate arbitration between these three $Q$-values is determined by a softmax choice rule ${ }^{40}$ with an additional lapse rate ${ }^{41}$.

In the model comparison (cf Supplementary Information), we found that the subjective costs per step followed a nonlinear, sigmoidal, function. This means the subjective costs of sampling were small initially but increased markedly as more information was gathered, similar to a previously described urgency signal ${ }^{36}$. This process is mainly controlled by an 'impatience' parameter $p$ that describes the stage at which these costs start escalating. The model comparison also revealed that subjects did not correctly represent the external costs in the decreasing condition, leading to suboptimal oversampling in this condition (cf Fig. 2, Supplementary Information). This is why the winning model absorbs both external and internal costs in the urgency signal.

\section{Model-based analyses}

To examine the cognitive mechanisms behind the group differences we fitted a set of recently developed models to each subject's data (computational model is detailed in Supplementary Information). Using the best fitting model (determined using Bayesian Information Criterion), we then compared the model predictions between the two groups. First, we compared decision thresholds so as to formalize any variation in information gathering behaviour. Decision thresholds are well known from passive evidence accumulation models that use a fixed stopping rule ${ }^{42,43}$. At each stage of information gathering, these thresholds characterise the mean difference in the evidence for the two colours at which subjects are willing to make a decision. In our task the threshold is influenced by the finiteness of the problem (i.e., that there are only 25 squares to sample and a majority of 13 cards is sufficient to be $100 \%$ certain) and the subjective cost of sampling, which reduces the $Q$-values for non-deciding (cf. Fig. S3). We used our computational model to compute the decision thresholds, computed as the mean evidence difference when a simulated agent (using the bestfitting subject-specific parameters) made a decision, separated for each sampling stage. With this measure we can assess how much evidence a (simulated) subject needs at each stage ${ }^{39}$, but also examine how a decision criterion might collapse as a function of sampling.

In our task, the collapsing decision threshold is driven by two factors: the finite horizon of the task, and a subjective urgency signal. The former factor is independent of subjects' preferences and captures the fact that as one gets close to opening all cards, a smaller evidence difference is needed to reach an absolute majority (i.e., 13 cards). The second factor, which we term urgency, is based on the observation that subjects express subjective costs of sampling information and these costs escalate as sampling proceeds. The stage when these costs escalate is determined by the impatience parameter $p$ (midpoint of a sigmoid cost function).

Group differences for decision thresholds and urgency signals were assessed using a cluster-extent permutation test ( $p<.05$, height threshold $t=1,1000$ iterations $)^{44}$.

To understand which aspects of the model were giving rise to the observed differences, we compared model parameters between groups using non-parametric Wilcoxon rank-sum tests, focusing on the impatience parameter $p$ in the fixed condition, which our previous work suggested was diagnostic for patients with $\mathrm{OCD}^{7}$.

\section{Results}

\section{Increased information gathering and winnings in high compulsive subjects}

We first asked whether subjects with high compulsive scores sought more information before making decisions 


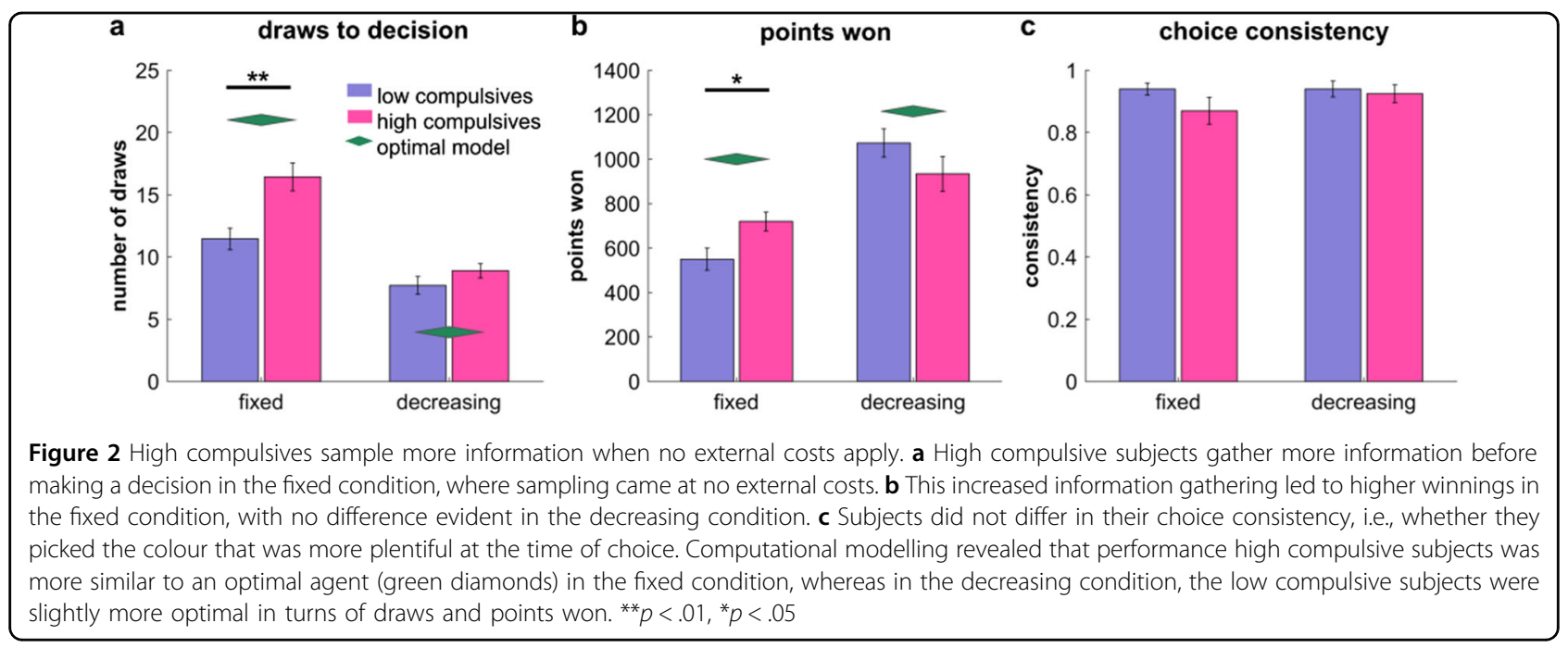

by comparing the number of cards they opened prior to a decision. We found a main effect of group $(F(1,38)=$ $10.15, p=.003, \eta_{p}^{2}=.211$ Fig. 2a) showing that high compulsives gathered more information before deciding, relative to the low compulsive group. An additional interaction effect $\left(F(1,38)=7.45, p=.010, \eta_{p}{ }^{2}=.164\right)$ demonstrates that the group difference was stronger in the fixed condition, in which no external cost for sampling was imposed, as confirmed by a significant effect in this condition $(t(38)=3.52, p=.001, \mathrm{~d}=1.11,95 \%$ confidence intervals $\mathrm{CI}$ : 2.1-7.9) but not in the decreasing condition $(t(38)=1.28, p=.207, \mathrm{~d}=.40, \mathrm{CI}:-.68-3.03)$ in which costs were imposed. The effect in the fixed condition also remained when adding depression (BDI total), anxiety (STAI trait and state) and IQ scores as covariates (multiple regression analysis, $t(34)=3.26, p$ $=.003$ ), thus ensuring that the difference was driven by the compulsivity characteristics of the groups. We also found that both groups gathered more information in the fixed condition than in the decreasing condition, evident in a main effect of condition $(F(1,38)=65.38, p<.001$, $\eta_{p}{ }^{2}=0.632$ ).

We next asked whether this increased sampling behaviour had a beneficial effect on outcomes, i.e. whether the high compulsive group won more points. A significant group-by-condition interaction $(F(1,38)=7.00, p=.012$, $\eta_{p}{ }^{2}=.156$, Fig. $\left.2 \mathrm{~b}\right)$, but no group main effect $(F(1,38)$ $\left.=.063, p=.803, \eta_{p}{ }^{2}=.002\right)$ indicated that subjects with high compulsivity scores won more points. However, this was only the case in the fixed condition, as confirmed in a direct comparison (fixed condition: $t(38)=2.60, p=.013$, $\mathrm{d}=.82$, CI: 37-302; decreasing condition: $t(38)=1.38, p$ $=.176, \mathrm{~d}=.44, \mathrm{CI}:-65-343)$. Again, the effect in the fixed condition remained when controlling for anxiety, depressive and IQ scores $(t(34)=2.71, p=.011)$. Additionally, a main effect of condition $(F(1,38)=39.93, p$ $\left.<.001, \eta_{p}{ }^{2}=.512\right)$ showed that both groups won more points in the decreasing condition, as would an optimal performing agent (cf. Fig. 2b).

To confirm that improved winnings reflected increased sampling, and not just less random behaviour, we examined whether subjects selected the colour that were more plentiful at the time of the decision (here termed choice consistency). Note that this does not directly translate into whether the subjects chose the colour that formed the overall majority, which in turn is directly reflected in the subjects' winnings. We found no evidence for a group difference $\left(F(1,38)=1.78, p=.190, \eta_{p}{ }^{2}=.045\right.$, Fig. 2c), nor a condition $\left(F(1,38)=.920, p=.344, \eta_{p}{ }^{2}=.024\right)$ or interaction effect $\left(F(1,38)=.920, p=.344, \eta_{p}{ }^{2}=.024\right)$. This indicates similar levels of randomness in both groups.

\section{Independent effects of intolerance of uncertainty and compulsivity on information gathering}

On a symptom level, excessive information gathering and indecisiveness have often been related to an intolerance of uncertainty and a pervasive perfectionism, especially in the context of $\mathrm{OCD}^{5,45}$. We thus obtained the subjects' self-reported intolerance of uncertainty (IU questionnaire $^{33}$ total score) and perfectionism (Frost Multidimensional Perfectionism Scale total; FMPS ${ }^{46}$ ). We observed a significant group difference in their intolerance of uncertainty $(t(38)=-2.05, p=.047$; Table 1$)$, but not in perfectionism $(t(38)=-.94, p=.353$; Table 1$)$. To assess whether IU and compulsivity independently explained the increased information gathering behaviour in the fixed condition, we assessed their independent contributions using a multiple regression. We found that both compulsivity $(t(37)=2.80, \quad p=.008)$ and selfreported intolerance of uncertainty $(t(37)=2.30, p$ $=.027$ ) predicted information gathering behaviour, 


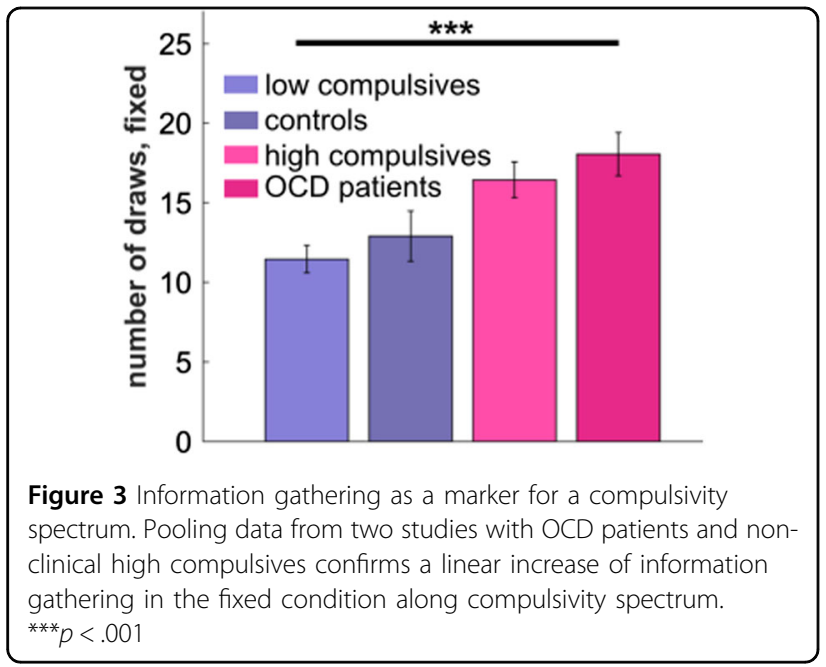

independently of each other (Fig. S6). No other measure (anxiety, depression, perfectionism, impulsivity, age, IQ) correlated with information gathering in the fixed condition (all $p$ 's $>.2$ ).

\section{Information gathering along the compulsivity spectrum}

Our finding of increased information gathering in the fixed condition in high compulsive subjects closely resembles our previous finding in adolescent OCD patients $^{7}$. A key prediction of a conception of compulsivity as a dimension would be that information gathering increases along the compulsivity spectrum, and where OCD patients would express the most extreme information gathering behaviour. We examined this prediction by pooling the two studies (previous OCD study and present study) and tested for a linear increase in information gathering across groups (low compulsives $<$ controls in OCD study [not selected for low compulsivity] $<$ high compulsives $<$ OCD patients). Here we found a highly significant effect of compulsivity group on information gathering (Fig. $3, t(70)=4.33, p<.001$ ), supporting the notion that information gathering is a general feature of a compulsivity spectrum.

\section{Increased decision threshold in high compulsives due to altered subjective urgency in fixed condition}

To understand the mechanisms and processes likely to drive the observed group difference in information gathering, we used a Bayesian computational model. Model comparison (Fig. S1) revealed that task behaviour of subjects was best described if we assume an urgency signal that rises in a nonlinearly manner during sampling and promotes choosing, independently of current evidence. This is implemented in our model in terms of subjective costs of sampling that escalate from an initially small value as sampling progresses, closely resembling urgency signals proposed for perceptual decision making ${ }^{36,37,47}$.

To formalize, and assess, how high and low compulsive subjects differ in their model predictions, we computed decision thresholds which reflected how much more evidence for one colour is needed to make a decision at each stage in the information sampling process (i.e., number of opened yellow-blue cards). Using model simulations, we found these thresholds collapsed over samples, in other words subjects became more liberal and were content to make a decision on weaker grounds. This collapse occurred significantly earlier for low compared to the high compulsive group in the fixed (Fig. 4a; $p=.024$, clusterextent correction), but not in the decreasing, condition ( $p$ $=.077$, cluster-extent correction).

One of the key drivers of the collapsing decision thresholds is an urgency signal, and in our model this is cast as subjective costs per step. In the fixed condition there are no extrinsic cost, so this urgency reflects purely intrinsic (energy, cognitive or time) costs for continuing to sample. In the decreasing condition, the best-fitting model encompasses the externally applied costs within a single term (see Supplementary Information for detailed analyses of different costing models), which is why these costs arise markedly earlier in the latter condition (Fig. 4b).

When analysing this urgency signal we found that it arose significantly earlier in the low compared to the high compulsive subjects in the fixed condition (Fig. 4b; fixed condition: $p=.004$, cluster-extent correction; decreasing condition: $p=.212$, cluster-extent correction).

Finally, we compared model parameters to probe group differences in decision thresholds further. We found a significant difference in the impatience parameter $p$ for the fixed condition $(z(311)=-2.66, p=.008$, Fig. S2), similar to what we observed in a previous study of diagnosed patients with $\mathrm{OCD}^{7}$. This parameter is a key determinant of when the urgency signal starts escalating. We did not observe any difference for other model parameters (cf. Fig. S2).

\section{Discussion}

Humans differ in how they negotiate speed-accuracy tradeoffs ${ }^{48-50}$. In sequential information gathering patients with OCD exhibit symptoms that can be construed as a distortion of this speed-accuracy tradeoff due to overweighting correct decisions ${ }^{6-13}$. Here we use computational modelling of the behaviour of a carefully curated sample of young people to demonstrate that excessive information gathering is a defining feature of an obsessive-compulsive spectrum, going beyond the clinical manifestation of OCD. 


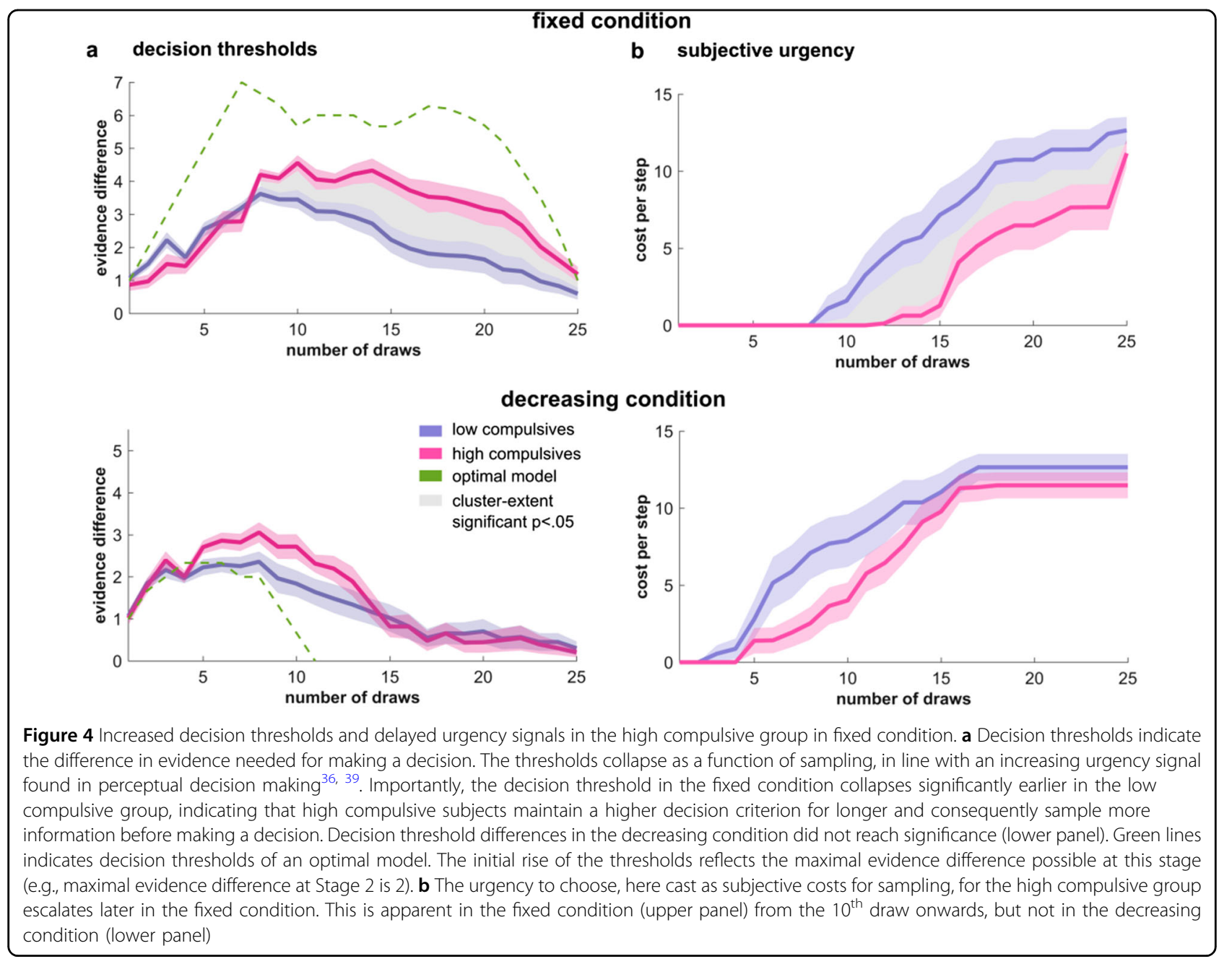

A pervasive indecisiveness and intolerance of uncertainty is seen as part of the core of OCD patients' obsessions and compulsions ${ }^{4,51}$. Such symptoms can be construed as an overweighting of the correctness of decisions, and an excessive investment in information gathering. Although such theories of compulsivity are venerable $^{52,53}$, behavioural analyses that test this idea are relatively scarce. In this study, we extend previous observations with OCD patients ${ }^{6-13}$ by showing that subjects with high, but non-clinical, symptoms of compulsivity exhibit a similar behavioural phenotype, albeit to an attenuated degree. Thus, both patients and non-clinical high compulsives manifest increased information gathering behaviour when there is no external cost for sampling. Notably this information gathering behaviour increased monotonically along a compulsivity spectrum, suggesting that increased information gathering generalises across a spectrum of neurodiversity. This includes, but is not confined to, clinical disorder at its extreme end. Interestingly, this increased sampling led to a better performance for subjects with high compulsivity scores in both studies, suggesting increased information gathering can be task beneficial (a feature rarely observed as consequence of a psychiatric symptom). Indeed, the fact that subjects with high compulsivity scores perform more proficiently on this task raises interesting questions, especially as indecisiveness is thought of as incapacitating $^{4,5,51}$.

We used computational modelling to probe the underlying cognitive mechanisms and revealed that this altered sampling behaviour in the fixed condition was due to an increased decision threshold in high compulsives or, more exactly, a slower collapse of the decision threshold across sampling. That is, the high compulsive group used a more restrictive decision criterion for a longer period. This slower collapse of a decision threshold was captured in our model as a delayed emergence of a subjective urgency to choose. Specifically, the impatience parameter that governs the stage at which this urgency grows most quickly is greater in high compulsive subjects, 
in accordance with our previous finding in patients with $\mathrm{OCD}^{7}$.

It is notable that high compulsives (as well as OCD patients as shown previously ${ }^{7}$ ) all have collapsing decision thresholds, with the onset of this collapse adapting with external task demands (i.e., for the fixed vs decreasing condition). This suggests that an elevated decision threshold is not an expression of cognitive inflexibility or rigidity, i.e. an inability to adjust strategies to maximise external rewards, but is the result of an arbitration between an internal need for certainty, and other internal and external demands, such as explicit sampling costs. Indeed, we found a group effect for information sampling in the fixed condition alone, and not in the decreasing condition (although differences in the reward structures in the two tasks that could also have contributed to this). The observation that high compulsive subjects had a lower reward rate (number of points per unit time, cf. Supplementary Information for analyses) than low compulsive subjects in the decreasing condition might suggest they were not as sensitive to this implicit metric. However, this reward rate did not differ between groups in the fixed condition. Thus although high compulsive subjects won more points overall, they did not win more points per unit time. This favours an interpretations that compulsive subjects solve a speed-accuracy tradeoff differently, favouring accuracy when there is no explicit costs for extended elaborations.

Considerations of the rate of reward prompt speculation as to whether the urgency signal is more directly linked to the time that has passed, the effort that has so far been exerted $^{54}$, or to the amount of information that has been obtained. As for most other sampling tasks, we lack the sensitivity to distinguish between these linked options. However, teasing these factors apart in future studies could shed light on whether the impatience that we observe is due to a perceived waste of sampling-time, a cognitive capacity issue due to an overwhelming amount of information, or some other additional factors.

Urgency signals to promote liberal decision making as a function of sampling have previously been described in perceptual decision making ${ }^{36,38}$. These signals are assumed to arise nonlinearly ${ }^{37,47}$, as we found, and to modulate evidence accumulation in the $\operatorname{brain}^{36,38,39}$. Given the relevance in compulsivity, it is interesting to make conjectures about the neural origins of this signal. One of the key areas described to modulate decision thresholds is the subthalamic nucleus $(\mathrm{STN})^{55-58}$, which has been found to be compromised in $\mathrm{OCD}^{59}$ and is a common target for deep brain stimulation in refractory OCD patients ${ }^{60,61}$. It is assumed that the STN closely communicates with areas of the medial prefrontal wall $^{55,62,63}$, such as the anterior cingulate cortex, which also has been found to be impaired in structure and function $^{64,65}$ and is another target area for OCD neurosurgery ${ }^{66}$.

It is interesting to conjecture how increased information gathering is related to other neurocognitive mechanisms that have been found as impaired in compulsivity, such as an excessive habitual behaviour ${ }^{16,67}$. It is possible that cognitive features such as inflexibility or a dominance of habitual modes of behaviour describe separate neurocognitive subtypes that express in similar compulsive symptoms. Alternatively, these features may arise at different stages in the evolution of what is often a chr

onic disorder, and where our findings pertain to an early phase as seen in our adolescent and young adult samples.

In our study, we observed that increased information gathering in the fixed condition did not load solely on one of the obsessive-compulsive subscales (cf. Supplementary Information). Rather, it loaded to some degree on most of the PI-WSUR subscales. This supports the notion that information gathering is not portraying a subtype on a symptom level (e.g., only compulsions or obsessions), but rather depicts a specific neurocognitive phenotype.

In this study, we used a targeted recruitment approach, where we carefully selected 40 subjects from a large population-based study. With a sample of 20 subjects per group, the group size was relatively modest and this was partly determined by our very selective recruitment and matching approach. We recruited subjects on the basis that they scored either high or low on a compulsivity spectrum, but who were otherwise matched on psychiatric dimensions such as anxiety or depression. This allowed us to study compulsivity independently of other, potentially confounding psychiatric traits and thus enabled us to attribute excessive information gathering more directly to compulsivity. Although modest in size our sample size is in line with previous patient studies that reported similar sample $\mathrm{e}^{7-11}$ and relatively large effect sizes $^{7,10}$.

Our study provides evidence for increased information gathering in conditions with no external costs, behaviour that is characteristic of a compulsivity spectrum, and that is seen in both non-clinical high compulsive subjects as well as in patients with OCD. This behaviour is driven by elevated decision thresholds and a slower emergence of an urgency to respond. By recruiting from a populationbased sample we control for potential confounders, such as other psychiatric dimensions, age or intellectual abilities. The findings lead us to propose that compulsivity is a phenotypic spectrum that is characterised by an increased need for certainty.

\section{Acknowledgements}

We thank Liz Harding, Danae Kokorikou, Hina Dadabhoy, Alex Hopkins, and Kalia Cleridou for helping with the recruiting. We thank Gita Prabhu for her support with the ethics application, recruiting and data organisation of this 
study. We also thank the NSPN Consortium for providing access to the UCHANGE database. The Wellcome Trust's Cambridge-UCL Mental Health and Neurosciences Network grant (095844/Z/11/Z) supported RJD, TUH and MM. RJD holds a Wellcome Trust Senior Investigator Award (098362/Z/12/Z). MM was also supported by the Biomedical Research Council. PD was supported by the Gatsby Charitable Foundation. The Max Planck UCL Centre is a joint initiative supported by UCL and the Max Planck Society. The Wellcome Trust Centre for Neuroimaging is supported by core funding from the Wellcome Trust (091593/Z/10/Z)

A complete list of the NSPN Consortium members can be found in the supplementary material.

\section{Author details}

${ }^{1}$ Wellcome Trust Centre for Neuroimaging, University College London, London WC1N 3BG, United Kingdom. ${ }^{2}$ Max Planck UCL Centre for Computational Psychiatry and Ageing Research, London WC1B 5EH, United Kingdom. ${ }^{3}$ Gatsby Computational Neuroscience Unit, University College London, London, United Kingdom

\section{Competing interest}

The authors declare that they have no conflict of interest.

\section{Publisher's note}

A complete list of the NSPN Consortium members can be found in the supplementary material.

\section{Supplementary information}

The online version of this article (doi:10.1038/s41398-017-0040-3) contains supplementary material.

Received: 24 March 2017 Revised: 24 August 2017 Accepted: 13 September 2017

Published online: 18 December 2017

\section{References}

1. Luce R. D. Response Times: Their Role in Inferring Elementary Mental Organization. OUP USA, 1986

2. Martin L. J., Müller G. E. Zur Analyse der Unterschiedsempfindlichkeit (Leipzig, 1899).

3. Woodworth R. S. Accuracy of voluntary movement. Psychol Rev Monogr Suppl (1899).

4. APA. Diagnostic and statistical manual of mental disorders: DSM-5 (American Psychiatric Association: Washington, DC, (2013)).

5. Tolin, D. F., Abramowitz, J. S., Brigidi, B. D. \& Foa, E. B. Intolerance of uncertainty in obsessive-compulsive disorder. J. Anxiety. Disord. 17, 233-242 (2003).

6. Fear, C. F. \& Healy, D. Probabilistic reasoning in obsessive-compulsive and delusional disorders. Psychol. Med. 27, 199-208 (1997).

7. Hauser, T. U. et al. Increased decision thresholds enhance information gathering performance in juvenile Obsessive-Compulsive Disorder (OCD). PLoS. Comput. Biol. 13, e1005440 (2017).

8. Pélissier, M.C. \& O'Connor, K. P. Deductive and inductive reasoning in obsessive-compulsive disorder. Br J Clin Psychol Br Psychol Soc 41, 15-27 (2002).

9. Volans, P. J. Styles of Decision-making and Probability Appraisal in Selected Obsessional and Phobic Patients. Br. J. Soc. Clin. Psychol. 15, 305-317 (1976).

10. Voon V. et al. Decisional impulsivity and the associative-limbic subthalamic nucleus in obsessive-compulsive disorder: stimulation and connectivity. Brain 140, 442-456 (2017)

11. Chamberlain, S. R. et al. A neuropsychological comparison of obsessivecompulsive disorder and trichotillomania. Neuropsychologia. 45, 654-662 (2007).

12. Grassi, G. et al. Think twice: Impulsivity and decision making in obsessivecompulsive disorder. J Behav Addict 4, 263-272 (2015).
13. Jacobsen, P., Freeman, D. \& Salkovskis, P. Reasoning bias and belief conviction in obsessive-compulsive disorder and delusions: jumping to conclusions across disorders? Br J Clin Psychol Br Psychol Soc 51, 84-99 (2012).

14. Casey, B. J. et al. DSM-5 and RDoC: progress in psychiatry research? Nat. Rev Neurosci. 14, 810-814 (2013).

15. Insel, T. R. The NIMH Research Domain Criteria (RDoC) Project: Precision Medicine for Psychiatry. Am. J. Psychiatry. 171, 395-397 (2014).

16. Gillan C. M., Kosinski M., Whelan R., Phelps E. A., Daw N. D. Characterizing a psychiatric symptom dimension related to deficits in goal-directed control. elife 2016; 5. doi:10.7554/eLife.11305.

17. Brown, T. A., Campbell, L. A., Lehman, C. L., Grisham, J. R. \& Mancill, R. B. Current and lifetime comorbidity of the DSM-IV anxiety and mood disorders in a large clinical sample. J. Abnorm. Psychol. 110, 585-599 (2001).

18. Vértes P. E. et al. Gene transcription profiles associated with inter-modular hubs and connection distance in human functional magnetic resonance imaging networks. Philos Trans R Soc Lond B Biol Sci 371 (2016); doi:10.1098/ rstb.2015.0362

19. Whitaker K. J. et al. Adolescence is associated with genomically patterned consolidation of the hubs of the human brain connectome. Proc Natl Acad SC USA. 113, 9105-9110 (2016).

20. Hauser T. U., Allen M., NSPN Consortium, Rees G., Dolan R. J. Metacognitive impairments extend perceptual decision making weaknesses in compulsivity. Sci. Rep. 7, 6614 (2017); doi:org/10.1038/s41598-017-06116-z.

21. Burns, G. L., Keortge, S. G., Formea, G. M. \& Sternberger, L. G. Revision of the Padua Inventory of obsessive compulsive disorder symptoms: Distinctions between worry, obsessions, and compulsions. Behav. Res. Ther. 34, 163-173 (1996).

22. Costello, E. J. \& Angold, A. Scales to assess child and adolescent depression: checklists, screens, and nets. J. Am. Acad. Child. Adolesc. Psychiatr. 27, 726-737 (1988).

23. Wood, A., Kroll, L., Moore, A. \& Harrington, R. Properties of the Mood and Feelings Questionnaire in Adolescent Psychiatric Outpatients: A Research Note. J. Child. Psychol. Psychiatr. 36, 327-334 (1995).

24. Reynolds, C. R. \& Richmond, B. O. What I think and feel: a revised measure of children's manifest anxiety. J. Abnorm. Child. Psychol. 6, 271-280 (1978).

25. Muris, P., Merckelbach, H., Ollendick, T., King, N. \& Bogie, N. Three traditional and three new childhood anxiety questionnaires: their reliability and validity in a normal adolescent sample. Behav. Res. Ther. 40, 753-772 (2002).

26. Spielberger, C. D., Gorusch, R. L., Lushene, R., Vagg, P. R. \& Jacobs, G. A. Manual for the State-Trait Anxiety Inventory. (Consulting Psychologists Press, Palo Alto, CA, 1983)

27. Beck, A. T., Steer, R. A. \& Brown, G. K. Manual for the Beck Depression Inventory-Il. (Psychological Corporation, San Antonio, TX, 1996).

28. Patton, J. H., Stanford, M. S. \& Barratt, E. S. Factor structure of the Barratt impulsiveness scale. J. Clin. Psychol. 51, 768-774 (1995).

29. Wechsler, D. Wechsler Abbreviated Scale of Intelligence. (The Psychologica Corporation: Harcourt Brace \& Company, New York, 1999).

30. Oldfield, R. C. The assessment and analysis of handedness: the Edinburgh inventory. Neuropsychologia. 9, 97-113 (1971).

31. Clark, L., Robbins, T. W., Ersche, K. D. \& Sahakian, B. J. Reflection impulsivity in current and former substance users. Biol. Psychiatry. 60, 515-522 (2006).

32. Clark L. et al. Validation of a novel measure of reflection-impulsivity for use in adult patient populations. J. Psychopharmacol. 2003.http://discovery.ucl.ac.uk/ 407900/ (accessed 4 Jul 2017).

33. Buhr, K. \& Dugas, M. J. The intolerance of uncertainty scale: psychometric properties of the English version. Behav. Res. Ther. 40, 931-945 (2002).

34. Moutoussis, M., Bentall, R. P., El-Deredy, W. \& Dayan, P. Bayesian modelling of Jumping-to-Conclusions bias in delusional patients. Cognit. Neuropsychiatry. 16, 422-447 (2011).

35. Watkins, C. J. C. H. \& Dayan, P. Q-learning. Mach Learn 8, 279-292 (1992).

36. Cisek, P., Puskas, G. A. \& El-Murr, S. Decisions in changing conditions: the urgency-gating model. J Neurosci Off J Soc Neurosci 29, 11560-11571 (2009).

37. Murphy, P. R., Boonstra, E. \& Nieuwenhuis, S. Global gain modulation generates time-dependent urgency during perceptual choice in humans. Nat Commun 7, 13526 (2016)

38. Thura, D., Beauregard-Racine, J., Fradet, C.-W. \& Cisek, P. Decision making by urgency gating: theory and experimental support. J. Neurophysiol. 108 2912-2930 (2012)

39. Thura, D. \& Cisek, P. Deliberation and commitment in the premotor and primary motor cortex during dynamic decision making. Neuron. 81, 1401-1416 (2014) 
40. Luce R. D. Individual choice behavior: a theoretical analysis (Wiley, 1959).

41. Guitart-Masip, M. et al. Go and no-go learning in reward and punishment: interactions between affect and effect. Neuroimage. 62, 154-166 (2012).

42. Ratcliff, R., Smith, P. L., Brown, S. D. \& McKoon, G. Diffusion Decision Model: Current Issues and History. Trends. Cogn. Sci. 20, 260-281 (2016).

43. Ratcliff, R. \& McKoon, G. The diffusion decision model: theory and data for twochoice decision tasks. Neural. Comput. 20, 873-922 (2008).

44. Hauser, T. U. et al. Temporally Dissociable Contributions of Human Medial Prefrontal Subregions to Reward-Guided Learning. J Neurosci Off J Soc Neurosci 35, 11209-11220 (2015).

45. Coles, M. E., Frost, R. O., Heimberg, R. G. \& Rhéaume, J. 'Not just right experiences': perfectionism, obsessive-compulsive features and general psychopathology. Behav. Res. Ther. 41, 681-700 (2003).

46. Frost, R. O., Marten, P., Lahart, C. \& Rosenblate, R. The dimensions of perfectionism. Cogn Ther Res 14, 449-468 (1990).

47. Drugowitsch, J., Moreno-Bote, R., Churchland, A. K., Shadlen, M. N. \& Pouget, A. The cost of accumulating evidence in perceptual decision making. J Neurosci Off J Soc Neurosci 32, 3612-3628 (2012).

48. Dickman, S. J. \& Meyer, D. E. Impulsivity and speed-accuracy tradeoffs in information processing. J. Pers. Soc. Psychol. 54, 274-290 (1988).

49. Johnson, D. R., Beasley, W. H. \& Bard, D. E. Abstract: A Multi-Level Model of Individual Differences in Speed/Accuracy Tradeoff (SATin). Multivar Behav Res 43, 654-655 (2008).

50. Lohman, D. F. The effect of speed-accuracy tradeoff on sex differences in mental rotation. Percept. Psychophys. 39, 427-436 (1986).

51. Goodman, W. K. et al. The Yale-Brown Obsessive Compulsive Scale. I. Development, use, and reliability. Arch. Gen. Psychiatry. 46, 1006-1011 (1989).

52. Esquirol J.-E.-D. Des maladies mentales considerées sous les rapports médical, hygiènique et médico-légal. Chez J.-B. Baillière, 1838.

53. Reed, G. F. The Obsessional-Compulsive Experience: A Phenomenological Reemphasis. Philos. Phenomenol. Res. 37, 381-385 (1977).

54. Hauser T. U., Eldar E., Dolan R. J. Separate mesocortical and mesolimbic pathways encode effort and reward learning signals. Proc Natl Acad Sci USA. 114, E7395-E7404 (2017); doi:https://doi.org/10.1073/pnas.1705643114.

55. Cavanagh, J. F. et al. Subthalamic nucleus stimulation reverses mediofrontal influence over decision threshold. Nat. Neurosci. 14, 1462-1467 (2011).
56. Green, N. et al. Reduction of influence of task difficulty on perceptual decision making by STN deep brain stimulation. Curr Biol 23, 1681-1684 (2013).

57. Coulthard, E. J. et al. Distinct roles of dopamine and subthalamic nucleus in learning and probabilistic decision making. Brain J Neurol 135, 3721-3734 (2012).

58. Ratcliff, R. \& Frank, M. J. Reinforcement-based decision making in corticostriatal circuits: mutual constraints by neurocomputational and diffusion models. Neural. Comput. 24, 1186-1229 (2012).

59. Welter, M.-L. et al. Basal ganglia dysfunction in OCD: subthalamic neuronal activity correlates with symptoms severity and predicts high-frequency stimulation efficacy. Transl Psychiatr 1, e5 (2011).

60. Greenberg, B. D., Rauch, S. L. \& Haber, S. N. Invasive circuitry-based neurotherapeutics: stereotactic ablation and deep brain stimulation for OCD. Neuropsychopharmacol Off Publ Am Coll Neuropsychopharmacol 35, 317-336 (2010).

61. Mallet, L. et al. Subthalamic nucleus stimulation in severe obsessivecompulsive disorder. N. Engl. J. Med. 359, 2121-2134 (2008).

62. Herz, D. M., Zavala, B. A., Bogacz, R. \& Brown, P. Neural Correlates of Decision Thresholds in the Human Subthalamic Nucleus. Curr Bio/ 26, 916-920 (2016).

63. Hauser T. U., Eldar E., Dolan R. J. Neural mechanisms of harm-avoidance learning: A model for obsessive-compulsive disorder? JAMA Psychiatry. 73, 1196-1197 (2016); doi:10.1001/jamapsychiatry.2016.1938.

64. Brem, S. et al. Neuroimaging of cognitive brain function in paediatric obsessive compulsive disorder: a review of literature and preliminary meta-analysis. $J$ Neural Transm Vienna Austria 119, 1425-1448 (2012).

65. Hauser T. U. et al. Increased fronto-striatal reward prediction errors moderate decision making in obsessive-compulsive disorder. Psychol. Med. 47, 1246-1258 (2017).

66. Dougherty, D. D. et al. Prospective long-term follow-up of 44 patients who received cingulotomy for treatment-refractory obsessive-compulsive disorder. Am. J. Psychiatr. 159, 269-275 (2002).

67. Gillan, C. M. et al. Disruption in the balance between goal-directed behavior and habit learning in obsessive-compulsive disorder. Am. J. Psychiatr. 168 718-726 (2011) 